\title{
Massive hemoptysis treated with embolization of an ectopic bronchial artery arising from the right thyrocervical trunk: a case report
}

Songhyon Cho ${ }^{1 *}$ D, Kenji Kubota ${ }^{1}$, Yoshikazu Hirose ${ }^{1}$, Norihiko Yoshimura ${ }^{2}$, Yui Murai ${ }^{3}$ and Yasuo Hirose ${ }^{1}$

\begin{abstract}
Background: Ectopic bronchial artery and non-bronchial systemic arteries may be the culprit vessels of hemoptysis. The main cause of clinical failure of bronchial artery embolization is incomplete embolization caused by the misidentification of the culprit arteries by conventional angiography. Multidetector computed tomography angiography is useful for visualizing the culprit arteries.

Case presentation: An 82-year-old man was admitted with hemoptysis. Preprocedural multidetector computed tomography angiography revealed an ectopic bronchial artery branching from the right thyrocervical trunk. Superselective embolization of the ectopic bronchial artery was performed using gelatin sponge particles and metallic coils. Hemoptysis was controlled by this procedure without any associated complications.

Conclusions: Ectopic bronchial arteries originating from the thyrocervical trunk are rare. Preprocedural multidetector computed tomography angiography is useful for visualizing the culprit arteries of hemoptysis, especially if a patient has an ectopic bronchial artery or an ectopic non-bronchial systemic artery.
\end{abstract}

Keywords: Hemoptysis, Embolization, Bronchial artery, Multidetector computed tomography, Computed tomography angiography

\section{Introduction}

Massive hemoptysis is one of the fatal respiratory symptoms and is most frequently bronchogenic. Bronchial artery embolization (BAE) is widely used to manage massive hemoptysis (Yoon et al. 2002). However, ectopic bronchial artery (BA) and non-bronchial systemic arteries (NBSAs), such as the subclavian, internal mammary, or inferior phrenic arteries, may be the culprit vessels of hemoptysis. The main cause of clinical failure of BAE is incomplete embolization caused by the misidentification of the culprit arteries by conventional angiography (Zhao et al. 2017). Multidetector computed tomography

\footnotetext{
* Correspondence: songhyon.cho.1105@gmail.com

${ }^{1}$ Department of Emergency and Critical Care Medical Center, Niigata City

General Hospital, 463-7, Shumoku, Niigata 950-1197, Japan

Full list of author information is available at the end of the article
}

(MDCT) angiography is useful for visualizing the ectopic origin of the BA and NBSAs during BAE that can be easily missed by conventional angiography ( $\mathrm{Li}$ et al. 2019). We report a rare case of an ectopic BA that originated from the right thyrocervical trunk. It was detected by preprocedural MDCT angiography and was completely embolized to control massive hemoptysis.

\section{Case presentation}

An 82-year-old man with hemoptysis was admitted to our hospital. He had a history of bronchiectasis, but this was the first time he had ever developed hemoptysis. He had first gone to a neighboring hospital and received antibiotics intravenously. He was referred to our hospital for further evaluation and management. Despite conservative management, including aggressive antimicrobial

\section{Springer Open}

(0) The Author(s). 2022 Open Access This article is licensed under a Creative Commons Attribution 4.0 International License, which permits use, sharing, adaptation, distribution and reproduction in any medium or format, as long as you give appropriate credit to the original author(s) and the source, provide a link to the Creative Commons licence, and indicate if changes were made. The images or other third party material in this article are included in the article's Creative Commons licence, unless indicated otherwise in a credit line to the material. If material is not included in the article's Creative Commons licence and your intended use is not permitted by statutory regulation or exceeds the permitted use, you will need to obtain permission directly from the copyright holder. To view a copy of this licence, visit http://creativecommons.org/licenses/by/4.0/. 


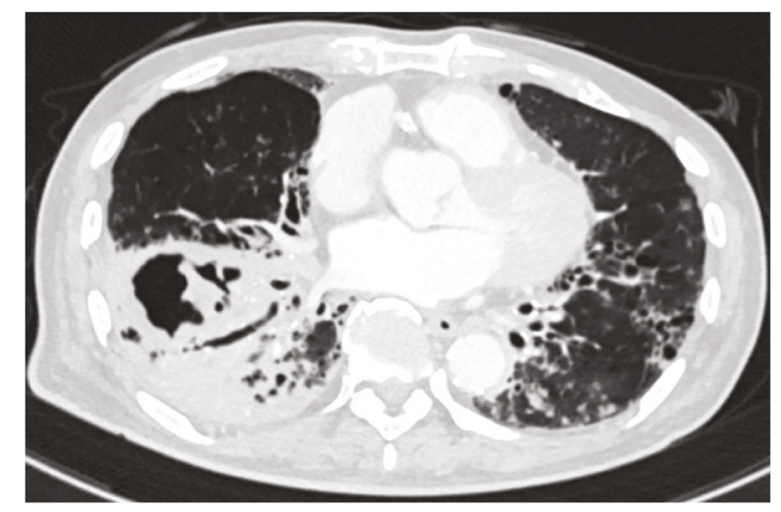

Fig. 1 The chest CT lung window imaging shows bilateral bronchiectasis and a large bulla with surrounding consolidation in the right lower lobe treatment, the patient experienced massive hemoptysis on the third day after admission and underwent emergency BAE.

Before emergency BAE, he underwent preprocedural evaluation by contrast-enhanced computed tomography (CT) scan with a 128-slice scanner (Siemens SOMATOM Drive, Siemens Healthineers, Tokyo, Japan), with the arterial phase $30 \mathrm{~s}$ after, and the delayed phase $90 \mathrm{~s}$ after intravenous contrast administration $(100 \mathrm{~mL}$ Oypalomin $370 \mathrm{mg} / \mathrm{mL}$, Fuji Pharma, Tokyo, Japan) at $3 \mathrm{~mL} / \mathrm{s}$. The chest CT lung window imaging showed bilateral bronchiectasis and a large bulla with surrounding consolidation in the right lower lobe, which was thought to be the source of hemoptysis (Fig. 1). Furthermore, computed tomography angiography revealed an ectopic BA that arose from the right thyrocervical trunk, supplying the right lower lobe in addition to the normal right BA (Fig. 2). This ectopic BA was significantly hypertrophied compared to the normal right $\mathrm{BA}$, suggesting that the ectopic BA was the culprit vessel of the hemoptysis (Fig. 3).

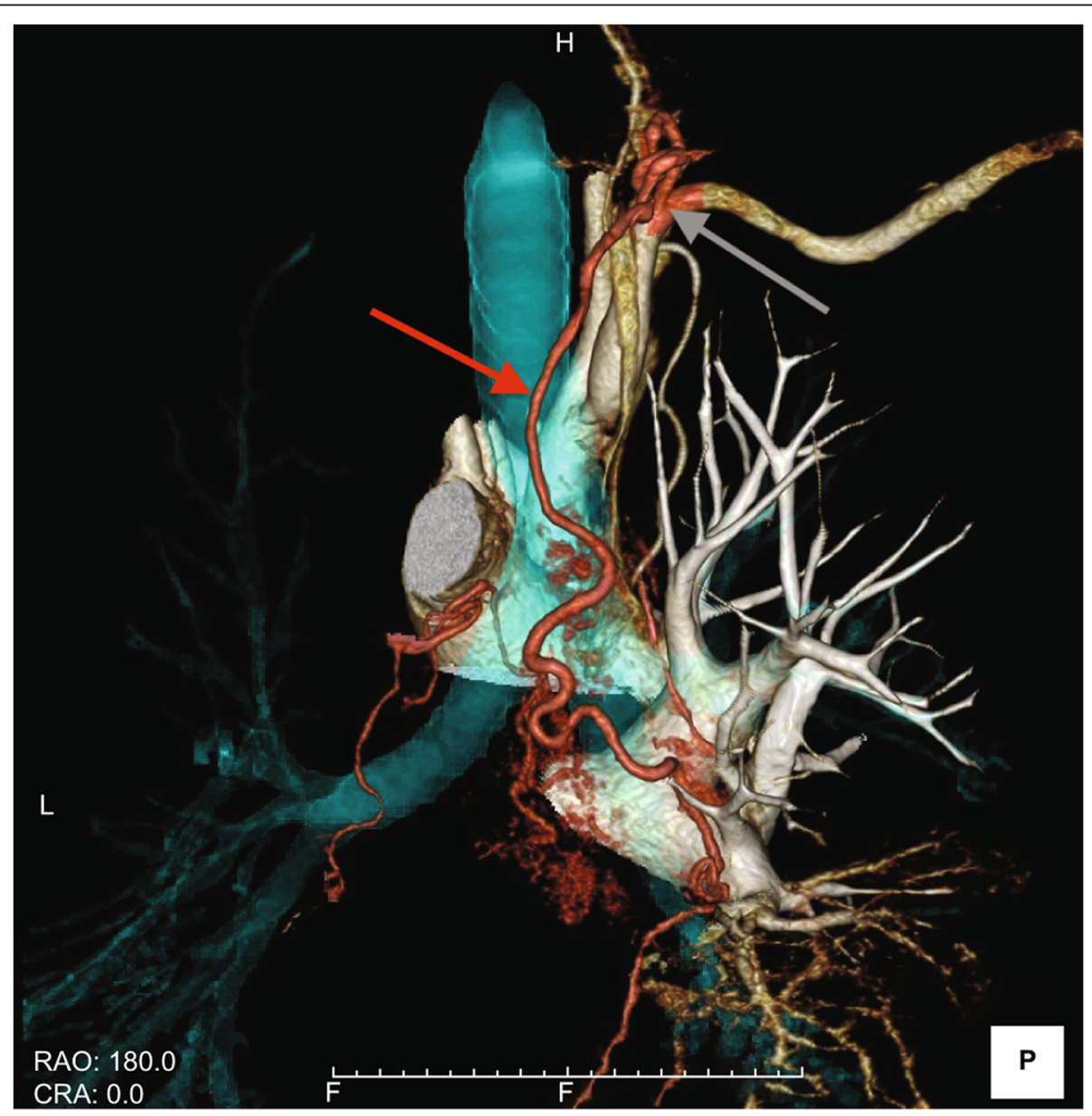

Fig. 2 Volume rendering reconstructed image of multidetector computed tomography angiography shows a hypertrophic ectopic right bronchial artery (red arrow) arising from the right thyrocervical trunk (gray arrow) 


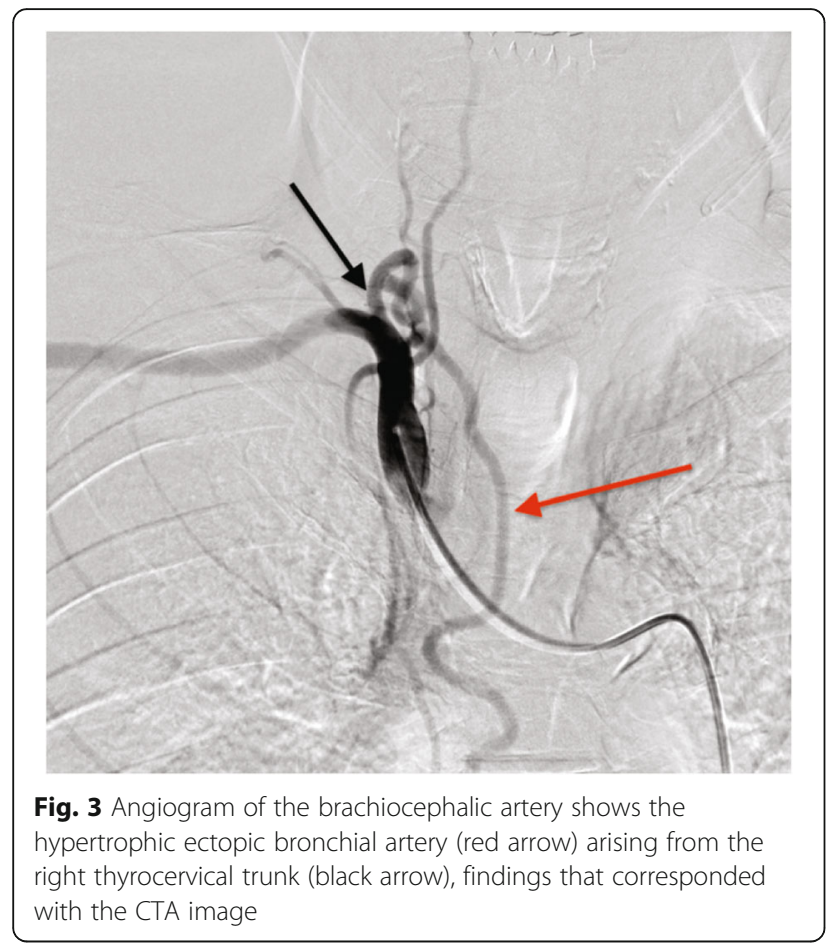

Superselective embolization of the ectopic BA was performed with gelatin sponge particles and metallic coils (Tornado, Cook Medical, Bloomington, USA) with a 1.9-2.9 Fr microcatheter (Breakthrough, Boston Scientific Japan, Tokyo, Japan) (Fig. 4A, B). After superselective embolization of the ectopic $\mathrm{BA}$, the normal right $\mathrm{BA}$ was selectively embolized with gelatin sponge particles (Fig. 5A, B).

Hemoptysis was controlled by this procedure without any procedural complications. He was discharged on day 5 of hospitalization with no complications.

\section{Discussion}

The BAs are the source of massive hemoptysis in $>90 \%$ of cases (Lorenz et al. 2012). Since the report published in 1973 by Remy et al., BAE has been revealed as an effective technique for the control of massive hemoptysis. However, interventional radiologists who perform BAE should keep in mind that the BA may show anatomical variations in terms of origin, branching pattern, and course (Yoon et al. 2002). Furthermore, a minority of massive hemoptysis result from NBSAs or pulmonary arteries (Lorenz et al. 2012).

BAs originating apart from between the $\mathrm{T} 5$ and $\mathrm{T} 6$ vertebrae are considered ectopic, and the incidence of ectopicity has been reported to range from $8.3 \%$ to 36\% (Michimoto et al. 2020). Ectopic BAs can be distinguished from NBSAs by their course along the major bronchi. On the other hand, NBSAs enter the pulmonary parenchyma through the adherent pleura or by way of the pulmonary ligament, and their course is not parallel to that of the bronchi (Sancho et al. 1998). In the present case, the branches of the right thyrocervical trunk ran along the main bronchus; therefore, this vessel was determined to be an ectopic BA rather than an NBSA.

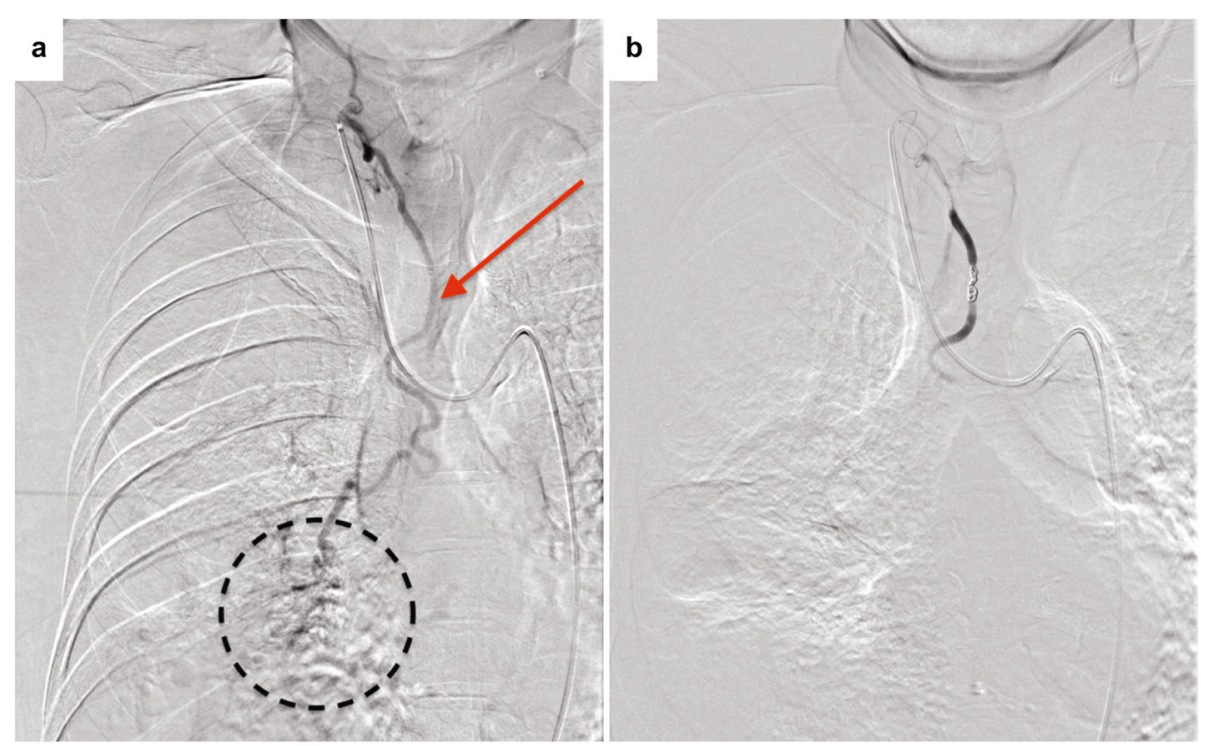

Fig. 4 A Selective angiogram of the ectopic right bronchial artery (arrow) shows parenchymal staining in the right lower lobe (dotted circle). B The parenchymal staining disappeared after embolization with gelatin sponge particles 


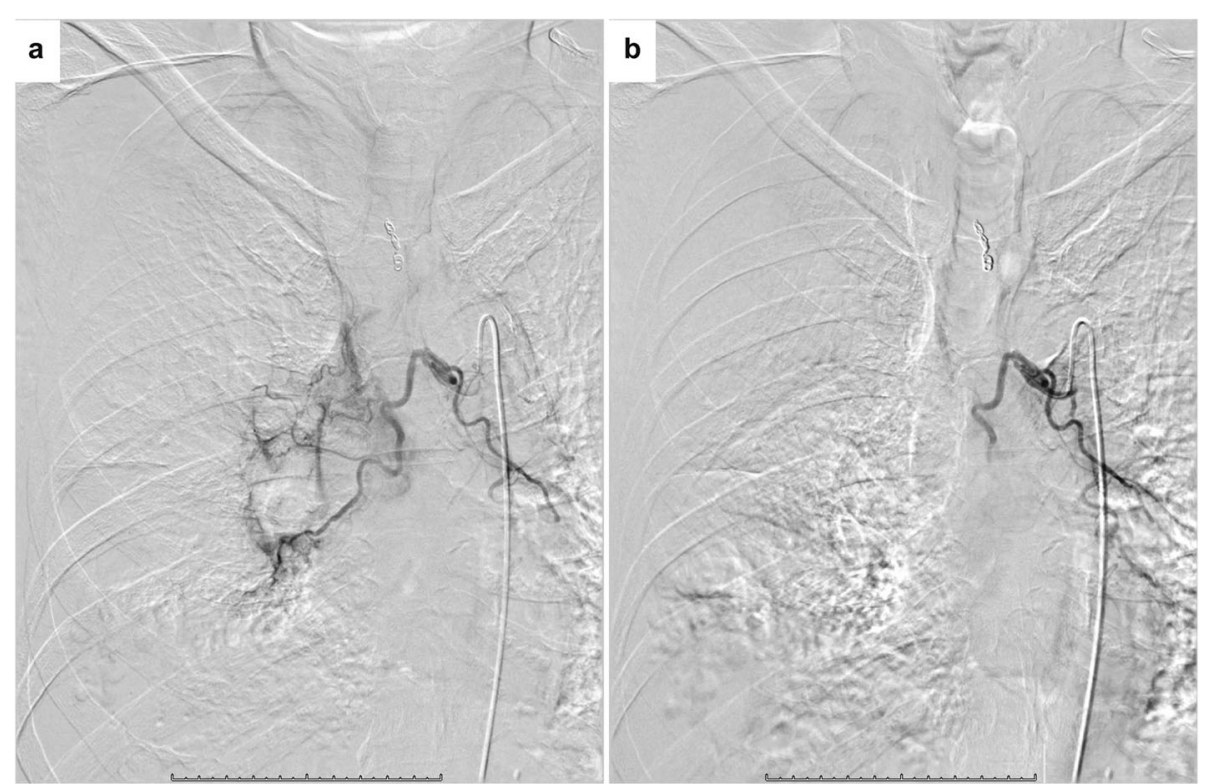

Fig. 5 A Selective angiogram of the common bronchial trunk shows normal bilateral bronchial arteries. B Only right BA was selectively embolized with gelatin sponge particles

Ectopic BAs originating from the thyrocervical trunk are rare. Summarizing several studies on MDCT angiography (Michimoto et al. 2020; Hartmann et al. 2007; Battal et al. 2011; Yener et al. 2015), only 9 of 624 patients (1.4\%) had BAs originating from the thyrocervical trunk. This rate was lower than those of the subclavian (29/ $624 ; 4.6 \%)$ or internal mammary $(11 / 624 ; 1.8 \%)$ artery origin. To our knowledge, most of the candidates for BAE for hemoptysis from a BA originating from the thyrocervical trunk have been reported to have a history of cystic fibrosis (Yoon et al. 2002; Hartmann et al. 2007; Battal et al. 2011; Yener et al. 2015). Furthermore, BAE was performed several times in these cases. In contrast, the patient in the present case had no history of cystic fibrosis and underwent $\mathrm{BAE}$ for the first time.

The rate of hemoptysis recurrence after $\mathrm{BAE}$ ranges from 9\%-55\% (Michimoto et al. 2020), and Zhao et al. reported that the main cause of clinical failure of BAE is incomplete embolization caused by misidentification of the culprit arteries by conventional angiography, especially for ectopic BAs and NBSAs (Zhao et al. 2017). A systematic review by Panda et al. (2017) reported that inadequate technique or incomplete embolization due to failure to detect all culprit arteries leads to early recurrence of hemoptysis within 3 months of BAE.

Many studies have reported that MDCT angiography is not only able to identify the source of bleeding and the underlying disease of hemoptysis, but also precisely detect the origins and courses of culprit arteries before $\mathrm{BAE}$, which is especially advantageous for visualizing the ectopic origin of BAs and NBSAs, which are easily missed by conventional angiography during BAE (Zhao et al. 2017; Li et al. 2019; Lorenz et al. 2012; Michimoto et al. 2020; Panda et al. 2017). Li et al. (2019) suggested that preprocedural MDCT angiography can reduce the recurrence rate of hemoptysis after BAE, and recommended MDCT angiography as a routine examination before BAE in patients with hemoptysis as far as possible. In the present case, visualization of the ectopic BA from the right thyrocervical trunk on preprocedural MDCT allowed us to perform a successful BAE. We recommend that preprocedural MDCT be performed so that the thyrocervical trunk can also be evaluated. In this case, the region proximal to the ectopic BA was occluded, the patient was discharged, and no rebleeding was observed. However, Yoon et al. (2002) argue that a coil should not be used for BAE because re-embolization is precluded if hemoptysis recurs. Therefore, there was an option to not use proximal coiling of the ectopic BA.

\section{Conclusion}

We performed BAE in a patient with an ectopic BA of the right thyrocervical trunk. Preprocedural MDCT angiography is useful for visualizing the culprit arteries of hemoptysis, especially if a patient has an ectopic origin of BAs or NBSAs.

\section{Abbreviations}

BAE: Bronchial artery embolization; BA: Bronchial artery; NBSAs: Nonbronchial systemic arteries; MDCT: Multi-detector computed tomography; CT: computed tomography

Acknowledgments

None. 


\section{Authors' contributions}

$\mathrm{SC}, \mathrm{KK}, \mathrm{YoH}$, and $\mathrm{YaH}$ performed the computed tomography angiography evaluations and developed a strategy for bronchial artery embolization. SC and $\mathrm{YOH}$ performed the treatment. YM performed pre- and post-procedure interventional follow-ups. SC, NY, and YaH drafted the manuscript. All authors read and approved the final manuscript.

\section{Funding}

Not applicable.

\section{Availability of data and materials}

Not applicable.

\section{Declarations}

Ethics approval and consent to participate

All procedures were in accordance with the ethical standards of the institution and with the 1964 Helsinki declaration. Informed consent was obtained from the patient in this case.

\section{Consent for publication}

Written informed consent was obtained from the patient for the publication of this case report and any accompanying images.

\section{Competing interests}

The authors declare that they have no competing interests.

\section{Author details}

'Department of Emergency and Critical Care Medical Center, Niigata City General Hospital, 463-7, Shumoku, Niigata 950-1197, Japan. ²Department of Radiology, Niigata City General Hospital, 463-7, Shumoku, Niigata 950-1197, Japan. ${ }^{3}$ Department of Respiratory Medicine, Niigata City General Hospital, 463-7, Shumoku, Niigata 950-1197, Japan.

Received: 7 October 2021 Accepted: 10 January 2022

Published online: 18 January 2022

\section{References}

Battal B, Akgun V, Karaman B, Bozlar U, Tasar M (2011) Normal anatomical features and variations of bronchial arteries: an analysis with 64-detector-row computed tomographic angiography. J Comput Assist Tomogr 35(2):253259. https://doi.org/10.1097/RCT.0b013e3182073c27

Hartmann IJ, Remy-Jardin M, Menchini L, Teisseire A, Khalil C, Remy J (2007) Ectopic origin of bronchial arteries: assessment with multidetector helical $\subset T$ angiography. Eur Radiol 17(8):1943-1953. https://doi.org/10.1007/s00330-0060576-8

Li PJ, Yu H, Wang Y, Jiang FM, Wang W, Li XO, Wang Y, Liang ZA (2019) Multidetector computed tomography angiography prior to bronchial artery embolization helps detect culprit ectopic bronchial arteries and nonbronchial systemic arteries originating from subclavian and internal mammary arteries and improve hemoptysis-free early survival rate in patients with hemoptysis. Eur Radiol 29(4):1950-1958. https://doi.org/10.1007/s00330018-5767-6

Lorenz J, Sheth D, Patel J (2012) Bronchial artery embolization. Semin Intervent Radiol 29(03):155-160. https://doi.org/10.1055/s-0032-1326923

Michimoto K, Takenaga S, Matsui Y, Enoki K, Nozawa Y, Higuchi T, Kano R, Kimura $\mathrm{T}$ (2020) Ectopic origin of bronchial arteries: still a potential pitfall in embolization. Surg Radiol Anat 42(11):1293-1298. https://doi.org/10.1007/ s00276-020-02495-7

Panda A, Bhalla AS, Goyal A (2017) Bronchial artery embolization in hemoptysis: a systematic review. Diagn Interv Radiol 23(4):307-317. https://doi.org/10.5152/ dir.2017.16454

Sancho C, Escalante E, Domínguez J, Vidal J, Lopez E, Valldeperas J, Montañá XJ (1998) Embolization of bronchial arteries of anomalous origin. Cardiovasc Intervent Radiol 21(4):300-304. https://doi.org/10.1007/s002709900265

Yener Ö, Türkvatan A, Yüce G, Yener AÜ (2015) The normal anatomy and variations of the bronchial arteries: evaluation with multidetector computed tomography. Can Assoc Radiol J 66(1):44-52. https://doi.org/10.1016/j.carj.2 014.07.001

Yoon W, Kim JK, Kim YH, Chung TW, Kang HK (2002) Bronchial and nonbronchial systemic artery embolization for life-threatening hemoptysis: a comprehensive review. RadioGraphics. 22(6):1395-1409. https://doi.org/1 $0.1148 / \mathrm{rg} .226015180$

Zhao T, Wang S, Zheng L, Jia Z, Yang Y, Wang W et al (2017) The Value of 320Row Multidetector CT bronchial arteriography in recurrent hemoptysis after failed transcatheter arterial embolization. J Vasc Interv Radiol 28:533-541.e1. https://doi.org/10.1016/j.jvir.2017.01.006

\section{Publisher's Note}

Springer Nature remains neutral with regard to jurisdictional claims in published maps and institutional affiliations.

\section{Submit your manuscript to a SpringerOpen ${ }^{\circ}$ journal and benefit from:}

- Convenient online submission

- Rigorous peer review

- Open access: articles freely available online

High visibility within the field

- Retaining the copyright to your article

Submit your next manuscript at $\boldsymbol{\nabla}$ springeropen.com 